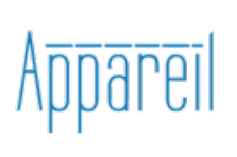

Appareil

$1 \mid 2008$

Le milieu des appareils

\title{
Appareils, messager, virus : pour une réhabilitation de la transmission
}

Sybille Krämer

\section{(2) OpenEdition}

1 Journals

\section{Édition électronique}

URL : http://journals.openedition.org/appareil/137

DOI : 10.4000/appareil. 137

ISSN : 2101-0714

Éditeur

MSH Paris Nord

Référence électronique

Sybille Krämer, «Appareils, messager, virus : pour une réhabilitation de la transmission », Appareil [En ligne], 1 | 2008, mis en ligne le 17 février 2008, consulté le 30 juillet 2020. URL : http:// journals.openedition.org/appareil/137; DOI : https://doi.org/10.4000/appareil.137

Ce document a été généré automatiquement le 30 juillet 2020.

\section{(c) (i) (3)}

Appareil est mis à disposition selon les termes de la Licence Creative Commons Attribution - Pas d'Utilisation Commerciale - Pas de Modification 4.0 International. 


\title{
Appareils, messager, virus : pour une réhabilitation de la transmission
}

\author{
Sybille Krämer
}

\section{Le principe postal et le principe érotique de la communication}

1 Le concept de "communication » mène une double vie dans le contexte théorique allemand. D'une part la théorie de la communication appelée pragmatique universelle à laquelle on associe le nom de Jürgen Habermas entend par communication un acte dialogique, un agir langagier réciproque, qui fonde entre les personnes une relation non instrumentale ${ }^{1}$. D'autre part la théorie de la communication fondée sur l'étude des médias et à laquelle on associe le nom de Friedrich Kittler comprend la communication comme un processus d'information fondé sur la transmission d'un message et le traitement de données au cours duquel différents médias interagissent en se renforçant mutuellement - quasiment sans avoir recours à l'Homme ${ }^{2}$. La conception fondée sur l'étude des médias se veut une alternative au concept personnel de communication, une déclaration de guerre à la communication visant un consensus et comprise comme fondement de la civilisation.

2 Mais curieusement ces deux concepts de communication convergent en un même point: ils ont en commun une tendance "apriorique», une volonté d'accéder aux «derniers fondements» des choses. Jürgen Habermas s'inscrit dans la tradition du tournant linguistique; pour lui, les actes de langage constituent la source de la raison et de la rationalité. Friedrich Kittler au contraire inaugure un ce que l'on a appelé en Allemagne un tournant médiatique; pour lui les médias sont le mécanisme et le champ magnétique de la dynamique culturelle. L'a priori médiatique a succédé à l'a priori linguistique. À l'autonomisation de la langue et de la communication correspond l'autonomisation des médias qui deviennent la condition indispensable de toute 
culture. De ce fait, il n'y a pas pour Kittler de « hors-média »; il défend une conception générative des médias: ils sont des instruments qui créent eux-mêmes ce qu'ils transmettent.

3 Ce qui nous mène à la question qui nous occupe : peut-on adopter l'idée porteuse de Kittler selon laquelle toute communication porte en elle une dimension médiatisante non éliminable, sans que les médias soient par là même élevés au rang de condition de possibilité de la culture ? Est-il donc possible de reconstruire le rôle des médias de manière à renoncer à poser les derniers fondements des choses? Peut-on développer une philosophie des médias sans adopter une conception générative des médias?

4 Pour être en mesure de donner une réponse positive à ces questions, il est nécessaire d'abandonner l'idée selon laquelle les médias seraient autonomes. De ce fait, nous procédons à un changement de perspective par rapport à la conception générative des médias et en outre aussi par rapport aux conceptions post-structuralistes, en formulant l'hypothèse suivante: Les médias sont hétéronomes, donc déterminés de l'extérieur et non autonomes ${ }^{3}$.

5 «Medium» signifie non seulement «moyen» mais aussi «milieu» et « intermédiaire ». Le tournant médiatique a surtout considéré ces derniers comme des moyens et des instruments. Mais qu'est-ce que cela signifie de les considérer avant tout comme « milieu » et comme " intermédiaire »? Confrontons encore une fois la théorie de la communication de la pragmatique universelle avec celle des techniques d'information.

6 (i) La théorie technique de la communication qui a été établie à l'origine par Shannon et Weaver part des instances de l'émetteur et du récepteur ${ }^{4}$. Son problème de départ est la distance qui les sépare. Le medium est donc ce qui se situe entre; il a pour fonction de transmettre et de maintenir à l'écart une «tierce personne importune». Cette transmission est asymétrique et unidirectionnelle. Le medium franchit une distance, mais sans aucunement l'annuler. Nous appellerons cela le principe postal de la communication.

7 (ii) Le modèle communicationnel proposé par la pragmatique universelle part en revanche de l'idée de la compréhension réciproque à travers des signes qui font sens. Son problème de départ est de coordonner en dissipant les désaccords ; la solution est de concevoir la communication comme un processus bipolaire et réciproque d'interaction sociale qui conduit au consensus. L'objectif de la communication n'est pas de lier mais de réunir. Du fait que la communication transforme l'hétérogénéité en homogénéité, la différence en identité, les participants se font en quelque sorte " un », ils parlent d'une même voix: nous appellerons cela le "principe érotique " de la communication.

8 Les différences entre le modèle postal et le modèle érotique sont évidentes : le principe postal conçoit la communication comme ce qui crée un lien sans pour autant annuler ni la distance ni l'éloignement. Il se réalise par la dissémination. Le principe érotique comprend la communication comme ce qui fait coïncider des éléments séparés, il oblitère la différence. Sa scène primitive, qui lui sert de modèle, est le dialogue. La place que ces deux conceptions réservent aux médias saurait difficilement être plus dissemblable. Dans la perspective postale, les médias sont indispensables pour remplir l'espace qui sépare l'émetteur du récepteur et pour rendre possible la transmission; dans la perspective érotique, les médias jouent un rôle perturbateur, dans la mesure où 
cette union d'éléments à l'origine séparés ne laisse (du moins en théorie) pas de place à un élément médian ou à un tiers.

9 Il ne fait aucun doute que la théorie de la communication défendue par la pragmatique universelle privilégie le dialogue et fait de lui le noyau indéracinable et la norme émancipatrice de notre communication, à l'aune de laquelle la dissémination apparaît comme une forme d'aliénation propre à la communication des médias de masse. Mais n'y a-t-il pas aussi une dimension tyrannique dans le fait d'hypostasier le dialogue en faisant de lui un modèle universel et l'ethos de la communication? Les innombrables pratiques sociales qui sont fondées sur l'utilisation de la langue tout en n'ayant aucun caractère dyadique, symétrique ou réciproque, ne s'en trouvent-elles pas stigmatisées et leur importance culturelle méconnue?

En effet bon nombre des pratiques de communication qui jouent un rôle fondateur pour les groupes et pour leur civilisation ne suivent justement pas le modèle imposé par la communication dialogique. La « communication érotique » propre à l'union d'éléments séparés constitue bien une manière de communiquer - du reste très séduisante -, mais vouloir l'élever au rang de forme absolue de la communication relève sans doute du romantisme.

\section{Socrate et Jésus : dialogue et/ou dissémination}

11 La tradition occidentale a donné naissance à deux symboles qui matérialisent la différence entre dialogue et dissémination ${ }^{5}$ : Socrate l'Athénien et Jésus de Nazareth ne constituent pas seulement un modèle pour la compréhension que l'occident a de la morale, mais ils incarnent aussi des modèles de communication opposés. Pour Socrate tel qu'il prend corps dans le Phèdre de Platon- le dialogue est une rencontre amoureuse, il est dirigé exactement vers son interlocuteur avec lequel une union en esprit est souhaitée. La rencontre dialogique devient un événement intime, dont l'étincelle et l'Eros se tariraient si un médium - l'écriture, par exemple - intervenait. Le comportement discursif de Jésus de Nazareth est tout autre. La parole de Jésus incarne le pôle antagoniste du dialogue socratique. Jésus se qualifie lui-même de semeur, qui répand des graines, dont certaines porteront des fruits et d'autres se dessècheront. Il s'agit donc de dissémination: un texte est transmis, qui ne s'adresse plus à quelques élus, mais à tous ceux qui "ont des oreilles pour entendre». Au mode ésotérique de communication réciproque, élaboré par Platon, qui s'adresse à quelques destinataires bien choisis et concentre la responsabilité sur celui qui parle, s'oppose le mode exotérique du discours asymétrique, incarné par Jésus, dans lequel la réciprocité est suspendue et où l'activité propre des récepteurs décidera de sa capacité à susciter l'adhésion.

12 Il ne s'agit pas ici de livrer une interprétation fidèle à la réalité historique. Ce que nous souhaitons, c'est d'une part relativiser la prétention - qui est aussi formulée par la philosophie - du modèle dialogique à rendre compte à lui seul d'une réalité et d'autre part réhabiliter le principe postal. Notre agir communicationnel n'a pas simplement un caractère disséminateur dans les situations d'éloignement. Même dans la rencontre en tête-à-tête - j'invoque ici Emmanuel Levinas ${ }^{6}$ - nous restons étrangers à notre interlocuteur. Reconnaître le principe postal signifie - là encore en référence à Levinas - renoncer à la prétention dialogico-tyrannique de l'union mentale et au nivellement égologique des différences de l'autre. 


\section{Le modèle du messager} médias dans le cadre de la communication sans avoir recours à une autonomisation et à une «transcendentalisation » des médias relevant d'une conception apriorique de ces derniers. Nous souhaitons à présent montrer l'hétéronomie constitutive des médias en exposant un «modèle du messager ».

consacrerons néanmoins une analyse car celle-ci pourrait constituer, dans le cadre de la théorie des médias, un modèle fécond pour développer les différentes significations du verbe « transmettre». créent un lien entre des choses séparées. Nous voudrions attirer l'attention sur sept aspects :

(1) Hétérogénéité : la communication implique la distance. La distance ne se réduit pas au domaine spatial mais prend également en compte la différence faisant que ceux qui communiquent restent étrangers l'un à l'autre, pris chacun dans leur histoire et dans leurs expériences différentes. La transmission d'un message implique la séparation non seulement Levinas, mais aussi Walter Benjamin et Jean-Luc Nancy nous l'ont rappelé. La réflexion portant sur le messager ne vise pas à expliquer seulement ce que sont la distance et la différence, mais aussi comment nous nous en accommodons. Notre hétérogénéité fait de notre rapport à la distance une - sinon LA - source de la civilisation.

(2) Hétéronomie : le messager n'est pas acteur et il ne s'est pas lui-même envoyé, il parle de la voix d'un autre. Le messager est donc bien celui qui ne parle pas en son nom propre. Il n'a pas même besoin de penser, de croire ni même de comprendre ce qu'il dit. L'impuissance discursive du messager constitue l'« empreinte négative » de la théorie des actes de langage. Il n'est pas étonnant que la philosophie soit née du refus du modèle du messager : la critique de Platon à l'endroit du rhapsode est toujours aussi une critique à l'endroit de celui qui conçoit son discours comme celui d'un messager.

(3) Rendre sensible l'invisible : Le caractère impropre du discours du messager renvoie au fait que ce dernier, en parlant, montre avant tout quelque chose. Dans le discours du messager le logos se transforme en aïsthésis et deixis. Le messager est une institution de la perception et de la visualisation; il rend visible ce qui n'est pas présent ou alors invisible. À l'horizon du modèle du messager, le fait de rendre perceptible, donc de montrer, devient la fonction fondamentale du dire.

(4) L'être-trois comme ferment de l'être-social : le messager fonde une relation sociale (et il faut entendre dans la "relation» le relaté, le compte-rendu). La position d'intermédiaire adoptée par le messager, qui est une tierce personne, inaugure une communauté de communication triadique, pour laquelle le messager est essentiel, sans pour autant en être le sujet. Les relations intersubjectives sont le plus souvent comprises comme des structures dyadiques comprenant un ego et un alter ego, un maître et un esclave, un émetteur et un récepteur; l'apparition d'un tiers apparaît comme une perturbation, un parasite. Pourtant, le passage au tiers ne permet-il pas d'atteindre un degré où les interactions sont en mesure de se muer en institutions? 
Dans son caractère social se niche en même temps la fragilité de l'institution du messager, ce qui le caractérise comme une figure du renversement: ce dernier se réalise quand le messager se départ de son hétéronomie et se pose comme autonome.

(5) La matérialité comme incarnation : quel que soit le message il doit pouvoir être séparé de son contexte de production et transporté. Le messager se meut dans l'espace intermédiaire du report de sens. L'extériorité de la signification est au fondement de son activité. Seule la séparation concrète du sens et du sensible, du texte et de la texture, de la forme et du contenu rend possible la transmission. La figure du messager rend palpable la matérialité de la communication.

21 (6) Neutralisation de soi : là où un message est envoyé, il y a toujours un enjeu : les messagers apportent soit le bonheur soit le malheur. Le messager pourtant reste toujours indifférent au contenu du message. Son lieu est le milieu : la neutralité est à la source de la fonction d'intermédiaire. Le "bon messager » fait preuve de réserve; il disparaît derrière son message. Le messager incarne le retrait dans l'accomplissement. C'est ce que nous rappelle le mythe du messager qui meurt, qui se consomme et se consume dans sa mission.

(7) Indifférence ontologique : le messager est impuissant sur le plan discursif, et il n'est donc pas étonnant qu'il puisse être remplacé sans difficulté dans sa fonction de transmission par des entités impersonnelles, des systèmes symboliques ou techniques. Rien ne se transmet aussi bien que la fonction de messager. Le messager est une personne qui se comporte comme si elle était une chose. Nulle part la coopération entre les hommes et les choses n'est aussi palpable que dans la mission du messager. La plupart du temps, des agrégats de choses et de personnes y ont un «statut d'acteur». Nous appellerons ces agrégats « Appareils».

Une réflexion complémentaire doit encore être menée: la conscience d'être un messager fait partie de la mission de ce dernier; mais il y a d'autre part dans l'institution $\mathrm{du}$ messager une remarquable dépersonnalisation, dédialogisation, désémantisation, bref : une dépotentialisation. Si nous radicalisons et extrapolons ces caractéristiques "réifiantes ", nous nous retrouvons confrontés au phénomène de la trace. De ce fait, notre exposition du modèle du messager reste incomplète tant que nous n'y incluons pas le «messager involontaire » qu'est la trace. Le fait même que la communication prenne sa source dans le fait de rendre sensible, trouve une expression claire dans ce que «nous montrent les traces ». Le messager et la trace se comportent comme les deux faces d'une même médaille.

La valeur heuristique du modèle du messager consiste pour nous dans le fait qu'elle apporte un éclairage conceptuel sur des phénomènes de transmission et sur diverses modalités de la médialité. La transmission est un phénomène ubiquitaire dont les champs d'application sont aussi divers que la société, la langue, la médecine, la psychanalyse, l'économie et la religion. Il est possible de traiter le modèle du messager comme le point zéro d'un système de coordonnées, dans lequel nous pouvons inscrire et décrire les différents phénomènes qui relèvent du " passage » rendu en français par le préfixe «trans- ». Nous ne livrons ici que quelques exemples : les anges transmettent la parole de Dieu, les virus transmettent des infections, en psychanalyse des types de sentiment sont transférés, l'argent transfère la propriété, les traductions transposent les langues, les témoins transmettent un savoir grâce à la confiance que l'on a en eux. À chacun de ces domaines correspond la logique fonctionnelle propre au medium développée à partir du modèle du messager : les anges transmettent du fait de leur 
hybridité, les virus par le biais de la transcription, le psychanalyste transfère par résonance affective, l'argent par l'indifférenciation, les traductions transposent par complémentarité et les témoins transmettent leur savoir grâce à leur crédibilité. Afin d'obtenir une représentation plus concrète de ces figures de la transmission, nous regarderons de plus près trois exemples : l'ange, le virus et le témoin.

\section{Les figures de la transmission (topoï de la transmission)}

(1) Ange - gr. (du Grec) "angelos»- signifie «messager». Les anges sont des constructions imaginaires qui, dans toutes les religions monothéistes, peuplent la solitude sculpturale de leur Dieu. La figure de l'ange, qui est en outre le motif le plus représenté de la peinture occidentale, donne une réponse à la question de savoir comment quelque chose peut être transmis entre deux éléments qui sont radicalement étrangers et très éloignés l'un de l'autre. L'astuce du "principe angélique » - Michel Serres et Andrei Plesu le soulignent ${ }^{7}-$, c'est l'hybridation : l'ange incarne aussi bien des qualités du divin que des qualités de l'humain, et ce non par un dépassement qui consisterait en une abolition au profit d'un tiers, mais dans la coexistence. Cette hybridité est atteignable seulement par une corporéité imaginaire. Bien que la doctrine angélique de la théologie souligne le plus souvent le caractère immatériel de l'ange, ce dernier ne peut être l'organe de transmission de Dieu que par la corporéité qui lui permet d'atteindre les hommes. De plus, l'ange se trouve à mi-chemin entre Dieu et l'homme: l'annulation de ce principe par Lucifer entraînera sa chute! Le diabolique constitue le revers de la figure de l'ange.

(2) La transmission de maladies par infection nous confronte à une logique de transmission d'une autre nature. Ici aussi le point de départ est une différence: l'« écart » existant entre un organisme infecté et un organisme non infecté, lequel est lui-même transformé en foyer infectieux par "l'invasion" d'un agent pathogène associé à un vecteur. La contagion n'est rendue possible que parce qu'il existe une différence entre l'étranger et le propre. C'est ce qu'utilise l'immunisation qui constitue une stratégie d'annulation de cette différence visant à rompre la chaîne de transmission de la maladie. Parmi les différentes formes de transmission de l'infection, le virus biologique est particulièrement intéressant. Les virus ne vivent pas, mais ils se reproduisent. Le principe du virus repose sur le fait qu'il entre dans la cellule et utilise les processus de réplication, de transcription et de translation de celle-ci pour la multiplication de son propre matériel génétique. La transmission par le virus est associée à une transcription. L'ADN de la cellule d'accueil est recodé dans l'ADN du virus. Le caractère de contrainte et la violence de la transmission de maladies a fait de l'infection un réservoir de métaphores pour la transmission culturelle : par exemple le caractère épidémique de la violence développé par René Girard ${ }^{8}$ ou la contagion des spectateurs à la vue d'une performance théâtrale ${ }^{9}$.

(3) Nous parvenons à notre dernière figure de la transmission, à savoir : le témoin. Les témoins agissent dans des situations d'incertitude ou d'ignorance, où il est nécessaire d'avoir recours à des témoignages pour établir la vérité sur un événement généralement lié à une violation du droit. Dans l'idéal, le témoin a assisté à la situation et ne tient pas son savoir que de la rumeur, il se doit de donner une expression verbale à la perception qui lui est propre : il doit «traduire » ce qu'il a vu en un discours, une 
expérience privée en un propos officiel. Un témoignage nécessite un auditoire, devant lequel on témoigne. La relation du témoin à l'auditoire est asymétrique : le témoin était présent lors d'un événement auquel l'auditoire n'a pas accès. De ce fait tout témoin porte en lui la possibilité du faux témoignage. Le fait de témoigner constitue la plupart du temps un acte de parole fortement institutionnalisé et donc performatif : le propos du témoin est vrai du fait de son statut. Néanmoins, sa vérité est liée à la crédibilité de la personne qui témoigne. C'est la confiance qui fait naître un savoir nouveau chez l'auditoire lors de la déposition. Le témoin n'est apte à transmettre son savoir que s'il est digne de confiance.

\section{La transmission donnant à voir}

Y a-t-il quelque chose qui se dessine au croisement de ces différents topoï de la transmission et qui serait commun à toutes ses formes, et donc essentiel pour la compréhension que nous en avons?

D'un point de vue très élémentaire, la transmission est une forme de rapport à la différence que l'on peut graduer, de sa forme la plus simple constituée par la distance spatio-temporelle jusqu'à sa forme la plus complexe, à savoir l'inaccessibilité réciproque des individus.

"L'attraction de la transmission » est déclenchée seulement par l'écart entre deux champs ou deux systèmes. Sans disparités, sans asymétrie, pas de transmission. Cette dernière suppose toujours la séparation et la division.

Mais si la transmission ne doit pas être comprise comme quelque chose qui nivelle cet écart et fait disparaitre la différence, comment est-il possible que le contact entre des systèmes différents préserve cette différence?

Nous avons atteint l'articulation de notre réflexion et souhaiterions revenir à une idée qui nous était déjà apparue comme une facette du modèle du messager. Conserver la différence dans la transmission signifie qu'il s'agit de rendre perceptible, de montrer. Nous devons penser la transmission comme un processus de création d'image, de visualisation. Le «logos» de la transmission consiste dans l'« aïsthésis », dans le fait de rendre perceptible, de donner à voir, de donner-une-image-de. Et « donner-une-imagede" signifie rendre visible l'irréductibilité d'une distance dans le champ de la perception. Car les images - c'est ce que, dans le contexte théorique allemand, nous rappelèrent Lambert Wiesing et Reinhardt Brandt - font apparaître quelque chose qui n'est cependant pas présent. Ce qui fait que les images sont plus que le signe de quelque chose, c'est qu'elles sont à la fois différentes et identiques à ce qu'elles représentent : les images représentent quelque chose qui est à la fois donné et non donné. En ce sens, les images montrent l'absence de ce qu'elles représentent, elles présentent dans ce qu'elles montrent une non-présence.

Peut-être le principe de la création d'image n'est-il jamais exprimé si clairement que dans la figure imaginaire de l'ange. Les anges n'existent que dans des images qui les représentent. Dans un curieux rapport à soi performatif, l'ange, en tant que messager de Dieu, réalise le principe de l'image - Massimo Cacciari l'a souligné ${ }^{10}$. Les anges rendent proche l'éloignement du divin, ils rendent l'invisibilité de Dieu visible dans leur propre apparition. 
$\mathrm{Ou}$, rapporté au cas du témoin: le témoin doit certes rendre par le discours une perception ou une expérience singulière, et donc se faire le "porte parole» d'un événement passé. Cependant la déposition du témoin n'est reconnue comme un discours vrai qu'à partir du moment où ce dernier peut en même temps prouver qu'il est digne de confiance. Mais ces qualités de sincérité et de crédibilité ne peuvent en aucune manière être formulées explicitement, elles ne peuvent qu'être montrées.

Tirons de cela un premier bilan : rendre perceptible constitue le cœur de la mission du témoin. Le principe postal, qui incarne une des dimensions de toute communication est en même temps un processus d' «aïsthétisation". Contre l'absolutisation de la communication qui la conçoit comme la disposition fondamentale de l'être-au-monde humain, issue de la discursivité, il faut souligner que le rendre-perceptible et le donnerà-voir constituent aussi le fondement de toute communication.

\section{La voix, l'écriture, l'ordinateur}

Le modèle du messager et de la visibilisation propre à la transmission permettent-ils de dégager des nouveaux horizons de médialité pour le concept de communication? Quelle signification ont nos réflexions pour des médias de communication comme la voix, l'écriture et l'ordinateur?

Habituellement, la voix est associée - au contraire de l'écriture qui passe pour la voie royale menant à une pensée éclairée - aux effets irrationnels de la parole. Derrida a de plus dénoncé la voix comme le point de cristallisation de la métaphysique de la présence propre à l'Occident ${ }^{11}$. Mais ces deux positions semblent ignorer le caractère hybride de la voix. Nietzsche signale cette hybridité en reconnaissant à la langue parlée aussi bien une dimension dionysiaque qu'une dimension apollinienne ${ }^{12}$. Dans la perspective dionysiaque, la voix se révèle être une caisse de résonance affective qui fonde dans le discours l'entente ou la discorde, indépendamment de son contenu argumentatif. L'entretien oral peut être comparé au fait de jouer de la musique à plusieurs ; il libère une force présémantique qui lie (et qui sépare à la fois), au-delà du sens et des convictions partagés. De plus, la voix est la trace du corps dans la parole indiquant dans la voix de poitrine une concrétisation de la puissance et dans la voix tremblante et défaillante l'impuissance subie. dimension durablement apollinienne. L'extériorisation de quelque chose d'intérieur dans l'extériorité du son n'ouvre absolument pas le chemin à une expérience de la pleine présence à soi comme Derrida le suggère, mais constitue - comme déjà Hegel et Humboldt l'ont montré - une forme de scission en nous-même qui crée « un monde des images mentales » dépassant le sujet, et grâce auquel nous pouvons agencer la fluidité de notre conscience originelle en une suite de pensées accessible à tous ${ }^{13}$.

Passons maintenant à l'écriture. Habituellement, l'écriture est considérée comme de la parole écrite, donc subordonnée sans autre forme de procès à l'ordre du discursif. Or nous devons concevoir le caractère hybride de l'écriture, entre langage et image, dans lequel des aspects discursifs et iconiques se mélangent depuis toujours. Les notations servent l'ordre typographique et topologique de l'espace dans la bidimensionalité d'une surface limitée qui sert de medium à la représentation. De ce fait, l'écriture est toujours aussi l'instrument d'une visualisation, elle possède d'ailleurs la caractéristique 
intéressante d'être ce qui crée matériellement ce qu'elle permet de visualiser. Pensons maintenant au chiffre zéro: il a été inventé pour servir de signe écrit qui marque l'absence d'un nombre dans l'expression décimale, il gagne ensuite, du fait du développement de la technique du calcul écrit, un droit de séjour dans l'espace des chiffres et devient enfin un signe pour un nombre défini, à savoir le nombre zéro : de "rien», on a fait "quelque chose». Mais cela s'explique seulement parce que le caractère sensible de l'écriture ouvre un champ d'action qui permet de faire usage des signes écrits ${ }^{14}$.

Pour terminer notre examen des médias en trois volets, encore un commentaire concernant l'ordinateur : longtemps l'ordinateur est passé soit pour un appareil visant à augmenter artificiellement l'intelligence, soit pour un medium de communication mondiale. Mais les prometteuses applications actuelles ne résident-elles pas précisément dans le fait "d'augmenter la visualisation»? Pensons par exemple à la « visualisation scientifique » et aux « moteurs de recherche ». La « scientific visualization » vit du fait que l'ordinateur rende accessible au domaine visuel des choses invisibles. Les images générées par ordinateur grâce aux nanotechnologies, par exemple, donnent une dimension visuelle à des impulsions produites par balayage électronique : elles rendent visible ce qui du fait de la longueur d'onde de la lumière perceptible est absolument invisible à l'œil nu. Et la simulation générée par ordinateur, qui a acquis le statut de troisième pratique scientifique, à côté de la construction théorique et de l'expérimentation, peut être interprétée comme une visualisation de théories.

41 Les moteurs de recherche d'internet nous confrontent à une toute autre dimension de la visualisation générée par ordinateur: car ce qu'ils font, c'est une cartographie adaptée à l'utilisateur des informations contenues par le réseau, qui transforme la multiplicité chaotique des pages internet en une diversité ordonnée qui permet de s'orienter efficacement, si bien que nous disposons d'une boussole pour pouvoir « naviguer sur cet océan d'informations ».

\section{Perspectives}

42 Une question pour finir : quelles conclusions doit-on tirer du modèle du messager pour la médialité de l'être-au-monde humain? Depuis la sécularisation amorcée depuis l'époque moderne, la représentation que l'homme se donne s'oriente sur les paradigmes de l'homo faber et de l'homo generator. Ces représentations de la créativité humaine sont eux-mêmes réglés sur un modèle démiurgique : l'homme est productif quand il crée et produit de manière autonome. La production en acquiert donc tout naturellement un statut primaire, tandis que la circulation est secondaire. Toutes nos conceptualisations de ce qu'est une machine s'orientent ainsi encore sur ce modèle de mécanismes génératifs.

43 Mais qu'est-ce alors que dire inversement que nous opérons plutôt comme des messagers et des intermédiaires que comme des démiurges? Que signifie le fait de définir la technique à l'horizon de fonctions de transmission et de médiation au lieu d'être fixé sur la création? Cela a-t-il un sens de définir le concept d'appareil à l'horizon d'activités de transmission?

44 Il paraît en tout cas impératif de réhabiliter la productivité des phénomènes de transmission. Considérer la culture dans une perspective de transmission nous fournit un espace qui nous autorise à porter un regard non génératif sur la condition humaine. 
Ce cadre repose sur quatre hypothèses : (premièrement) Les médias aïsthétisent : de ce fait la perception est revalorisée par rapport à la communication. (deuxièmement) Les médias nous permettent d'avoir un rapport à la différence, en créant un lien entre des choses hétérogènes sans annuler par là même l'hétérogénéité. (troisièmement) La transmission constitue un noyau de pratiques culturelles, si bien que le principe postal de la dissémination asymétrique doit être revalorisé par rapport à la prétention à l'absolu affichée par le dialogue. (quatrièmement) Notre force créatrice est à différents égards une productivité non démiurgique, qui trouve sa forme spécifique de créativité dans l'alliage des éléments hétérogènes, instituant par là même un autre rapport à la différence.

\section{BIBLIOGRAPHIE}

Cacciari Massimo, L'angelo necessario, Milan, Adelphi, 1986.

Derrida Jacques, La voix et le phénomène: Introduction au problème du signe dans la phénoménologie de Husserl, Paris, PUF, 1967.

Fischer-Lichte Erika, « Zuschauen als Ansteckung », in Mirjam Schaub, Nicola Suthor, Erika Fischer-Lichte (eds.), Ansteckung. Zur Körperlichkeit eines ästhetischen Prinzips, Munich, 2005.

Girard René, La violence et le sacré, Paris Grasset, 1972.

Habermas Jürgen, Théorie de l'agir communicationel, Paris, Fayard, 1987.

Kittler Friedrich, Draculas Vermächtnis: Technische Schriften, Leipzig, Reclam, 1993.

Krämer Sybille, « Die Heteronomie der Medien. Versuch einer Metaphysik der Medialität im Ausgang vom Botenmodell », Journal Phänomenologie, n 22, 2004, p. 18-38.

Krämer Sybille, « Operationsraum Schrift: Über einen Perspektivenwechsel in der Betrachtung der Schrift », in Schrift. Kulturtechnik zwischen Auge, Hand und Maschine, Munich, 2005.

Krämer Sybille, « Die "Rehabilitierung der Stimme". Über die Oralität hinaus », in Stimme, Doris Kolesch et Sybille Krämer (eds), Francfort-sur-le-Main, Suhrkamp, 2006.

Levinas Emmanuel, Totalité et infini. Essai sur l'extériorité, Den Haag, M. Nijhoff, 1961.

Levinas Emmanuel, Humanisme de l'autre homme, Montpellier, Fata Morgana, 1972.

Peters John Durham, Speaking into the air. A history of the idea of communication, Chicago/Londres, University of Chicago Press, 1999.

Plesu Andrei, Actualité des anges, Paris, Buchet-Chastel, 2005 (Despre ingeri, Bukarest, 2003).

Serres Michel, La légende des anges, Paris, Flammarion, 1993.

Weaver Warren, Shannon Claude E., Théorie mathématique de la communication, Paris, C.E.P.L., 1975. 


\section{NOTES}

1. Jürgen Habermas, Théorie de l'agir communicationel, Paris, Fayard, 1987.

2. Friedrich Kittler, Draculas Vermächtnis: Technische Schriften, Leipzig, Reclam, 1993.

3. Sybille Krämer, «Die Heteronomie der Medien. Versuch einer Metaphysik der Medialität im Ausgang vom Botenmodell », Journal Phänomenologie, $\mathrm{n}^{\circ} 22$, 2004, p. 18-38

4. Warren Weaver, Claude E. Shannon, Théorie mathématique de la communication, Paris, C.E.P.L., 1975.

5. John Durham Peters, Speaking into the air. A history of the idea of communication, Chicago/Londres, University of Chicago Press, 1999, 33ff.

6. Emmanuel Levinas, Totalité et infini. Essai sur l'extériorité, Den Haag, M. Nijhoff, 1961; Emmanuel Levinas, Humanisme de l'autre homme, Montpellier, Fata Morgana, 1972.

7. Michel Serres, La légende des anges, Paris, Flammarion, 1993, p. 171 ; Andrei Plesu, Actualité des anges, Paris, Buchet-Chastel, 2005 (Despre ingeri, Bukarest, 2003), 17ff.

8. René Girard, La violence et le sacré, Paris Grasset, 1972.

9. Erika Fischer-Lichte, "Zuschauen als Ansteckung", in Mirjam Schaub, Nicola Suthor, Erika Fischer-Lichte (eds.), Ansteckung. Zur Körperlichkeit eines ästhetischen Prinzips, Munich, 2005, $35 \mathrm{ff}$.

10. Massimo Cacciari, L'angelo necessario, Milan, Adelphi, 1986.

11. Jacques Derrida, La voix et le phénomène: Introduction au problème du signe dans la phénoménologie de Husserl, Paris, PUF, 1967.

12. Friedrich Nietzsche, Sämtliche Werke, Kritische Studienausgabe, hg. v. Giorgio Colli und Mazzino Montinari, Bd. 7, 360.

13. Sybille Krämer, «Die "Rehabilitierung der Stimme". Über die Oralität hinaus », in Stimme, ed. de Doris Kolesch et Sybille Krämer, Francfort-sur-le-Main, Suhrkamp, 2006, $269 \mathrm{ff}$.

14. Sybille Krämer, «Operationsraum Schrift: Über einen Perspektivenwechsel in der Betrachtung der Schrift ", in Schrift. Kulturtechnik zwischen Auge, Hand und Maschine, Munich, 2005, 23ff.

\section{AUTEUR \\ SYBILLE KRÄMER}

Université Libre de Berlin, Wissenschaftskolleg 